\title{
OUTPUT FEEDBACK CONTROL WITH CONSTRAINTS FOR NONLINEAR SYSTEMS VIA PIECEWISE QUADRATIC OPTIMIZATION
}

\author{
Nguyen Doan Phuoc ${ }^{1, *}$, Pham Van Hung ${ }^{2}$, Hoang Duc Quynh ${ }^{3}$ \\ ${ }^{1}$ Hanoi University of Science and Technology, 1 Dai Co Viet, Hanoi \\ ${ }^{2}$ Hanoi University of Industry, 298 Cau Dien, Hanoi \\ ${ }^{3}$ Thai Nguyen Industrial Economic-Technology College, Trung Thanh, Pho Yen, Thai Nguyen \\ *Email: phuoc.nguyendoan899@gmail.com
}

Received: 13 July 2016; Accepted for publication: 8 February 2017

\begin{abstract}
Very few output feedback control methods can be applied for a large class of nonlinear objects. If the control problem has supplementary constraints to satisfy, the number of suitable methods will be fewer. The paper proposes a nonlinear control method, which can be applied to output tracking control a wide range of various perturbed nonlinear objects. This output feedback controller is established based on piecewise quadratic optimizing subjected to input constraints for state feedback control and then combined with an appropriate EKF or UKF for system state observation. The simulation results obtained by applying this proposed controller to output tracking control inverted pendulum and boiler-turbine unit had confirmed its promising applicability in practice.
\end{abstract}

Keywords: quadratic optimization, EKF/UKF filtering, optimal control.

\section{INTRODUCTION}

Whereas almost nonlinear control methods are unable to handle unavoidable system constraints, the MPC method proposed in [1] seems to be a good controller for such constrained control problem. However, since the directly using nonlinear model for output prediction, this technique for NMPC requires additionally a penalty function for objective function in order to guarantee the stability of the closed system [2]. Unfortunately, the question how to choose this penalty function suitably is still open.

To overcome this circumstance, the piecewise linearization of nonlinear model on time axis for system output prediction looks to be a promising solution. The realization of this idea to predict outputs of a nonlinear system and then to establish completely an integral state feedback model predictive controller is the main content of the paper. Afterward, the obtained integral state feedback controller will be converted to an appropriate output feedback one based on 
separation principle by using an EKF or UKF additionally in order to determine all immeasurable system states.

\section{CONTROLLER DESIGN}

Consider a nonlinear system:

$$
\underline{x}_{k+1}=\underline{f}\left(\underline{x}_{k}, \underline{u}_{k}, \underline{\zeta}_{k}\right) \text { and } \underline{y}_{k}=\underline{g}\left(\underline{x}_{k}, \underline{u}_{k}, \underline{\xi}_{k}\right)+\underline{d}_{k}
$$

where both functions $\underline{f}(\cdot), \underline{g}(\cdot)$ are assumed to be smooth in $\underline{x}_{k}$ and $\underline{u}_{k}$,

$$
\underline{x}_{k}=\left(x_{1}[k], \ldots, x_{n}[k]\right)^{T}, \underline{u}_{k}=\left(u_{1}[k], \ldots, u_{m}[k]\right)^{T}, \underline{y}_{k}=\left(y_{1}[k], \ldots, y_{r}[k]\right)^{T}
$$

are the vector of all system states, vectors of inputs and outputs signals respectively at the current time instant $t_{k}=k T$, where $T$ is the sampling time, $\underline{\boldsymbol{S}}_{k}, \underline{\boldsymbol{\xi}}_{k}$ are white noises, which could propagate nonlinearity in system, and $\underline{d}_{k}$ is a vector of slow disturbances, which can be seen obviously as the model errors.

The here regarded control problem for the given nonlinear system (1) above is an output feedback controller $\underline{u}_{k}\left(\underline{x}_{k}\right)$ to design, which is subjected to the given constraint $\underline{u}_{k} \in U \subset \mathbb{R}^{m}$, so that its output vector $\underline{y}_{k}$ will be convergence asymptotically to any desired output vector $\underline{w}_{k}$, and this tracking control performance will not be affected by white noises $\underline{\underline{\varsigma}}_{k}$, $\underline{\xi}_{k}$ and by system errors $\underline{d}_{k}$.

\subsection{Receding horizon LTI predictive model}

Firsly, if all noises $\underline{\zeta}_{k}, \underline{\xi}_{k}$ and disturbance $\underline{d}_{k}$ in (1) are assumed to be negligeable, then from (1) the corresponding nominal model is obtained:

$$
\underline{x}_{k+1}=\underline{f}\left(\underline{x}_{k}, \underline{u}_{k}\right) \text { and } \underline{y}_{k}=\underline{g}\left(\underline{x}_{k}, \underline{u}_{k}\right)
$$

Since the smooth property, both function vectors $\underline{f}(\cdot), \underline{g}(\cdot)$ of the nominal model (2) can be now approximated at the previous time instant $t_{k-1}$ and during a short time interval $\left[t_{k-1}, t_{k}\right.$ ) afterwards as follows:

$$
\begin{aligned}
\underline{f}\left(\underline{x}_{k}, \underline{u}_{k}\right) & \approx \underline{f}\left(\underline{x}_{k-1}, \underline{u}_{k-1}\right)+\left.\frac{\partial \underline{f}}{\partial \underline{x}}\right|_{\underline{x}_{k-1}, \underline{u}_{k-1}}\left(\underline{x}_{k}-\underline{x}_{k-1}\right)+\left.\frac{\partial \underline{f}}{\partial \underline{u}}\right|_{\underline{x}_{k-1}, \underline{u}_{k-1}}\left(\underline{u}_{k}-\underline{u}_{k-1}\right) \\
& =\underline{x}_{k}+A_{k}\left(\underline{x}_{k}-\underline{x}_{k-1}\right)+B_{k}\left(\underline{u}_{k}-\underline{u}_{k-1}\right) \\
& =A_{k} \underline{x}_{k}+B_{k} \underline{u}_{k}+\underline{\varsigma}_{k} \\
\underline{g}\left(\underline{x}_{k}, \underline{u}_{k}\right) & \left.\approx \underline{g}\left(\underline{x}_{k-1}, \underline{u}_{k-1}\right)+\left.\frac{\partial \underline{g}}{\partial \underline{x}}\right|_{\underline{x}_{k-1}, \underline{u}_{k-1}} \quad\left(\underline{x}_{k}-\underline{x}_{k-1}\right)+\left.\frac{\partial \underline{g}}{\partial \underline{u}^{\prime}}\right|_{\underline{x}_{k-1}, \underline{u}_{k-1}} \quad \underline{u}_{k}-\underline{u}_{k-1}\right) \\
& =\underline{y}_{k-1}+C_{k}\left(\underline{x}_{k}-\underline{x}_{k-1}\right)+D_{k}\left(\underline{u}_{k}-\underline{u}_{k-1}\right)=C_{k} \underline{x}_{k}+D_{k} \underline{u}_{k}+\underline{v}_{k}
\end{aligned}
$$

where 


$$
\begin{aligned}
& A_{k}=\left.\frac{\partial \underline{f}}{\partial \underline{x}}\right|_{\underline{x}_{k-1}, \underline{u}_{k-1}}, \quad B_{k}=\left.\frac{\partial \underline{f}}{\partial \underline{u}}\right|_{\underline{x}_{k-1}, \underline{u}_{k-1}}, C_{k}=\left.\frac{\partial \underline{g}}{\partial \underline{x}}\right|_{\underline{x}_{k-1}, \underline{u}_{k-1}}, D_{k}=\left.\frac{\partial \underline{g}}{\partial \underline{u}}\right|_{\underline{x}_{k-1}, \underline{u}_{k-1}} \\
& \underline{\boldsymbol{\varsigma}}_{k}=\underline{x}_{k}-A_{k} \underline{x}_{k-1}-B_{k} \underline{u}_{k-1} \text { and } \underline{v}_{k}=\underline{y}_{k-1}-C_{k} \underline{x}_{k-1}-D_{k} \underline{u}_{k-1}
\end{aligned}
$$

are all now determined at the current time instant $k$. This implies finally a linear approximation along time axis of the nominal model (1) as follows:

$$
H_{k}:\left\{\begin{array}{l}
\underline{x}_{k+1}=A_{k} \underline{x}_{k}+B_{k} \underline{u}_{k}+\underline{\boldsymbol{\varsigma}}_{k} \\
\underline{y}_{k}=C_{k} \underline{x}_{k}+D_{k} \underline{u}_{k}+\underline{v}_{k} \text { for } t_{k} \leq t<t_{k+1}
\end{array}\right.
$$

This model $H_{k}$ will be used hereafter for the prediction of system outputs $\underline{y}_{k+i}$ in the current prediction horizon $1 \leq i \leq N$ as exhibited in Fig.1.

Figure 1. Receding predictive LTI model.

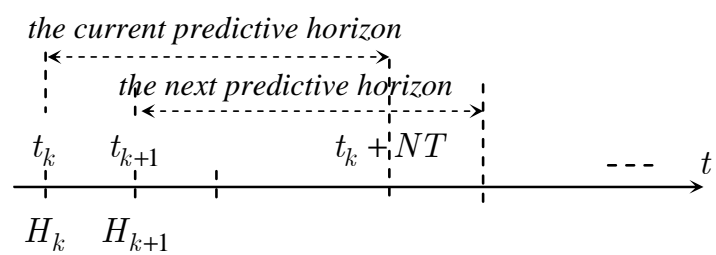

\subsection{Integral state feedback controller}

At the current time instant $k$ and based on the already measured system states $\underline{x}_{k}$ as well as the assumption that $\underline{\varsigma}_{k}, \underline{v}_{k}$ are constant during the current predictive horizon, all predictive system states $\underline{x}_{k+i}, 1 \leq i \leq N$ can be now obtained from the LTI predictive model (4) as follows:

$$
\begin{aligned}
\underline{x}_{k+i} & =A_{k} \underline{x}_{k+i-1}+B_{k} \underline{u}_{k+i-1}=A_{k}\left(A_{k} \underline{x}_{k+i-2}+B_{k} \underline{u}_{k+i-2}+\underline{\varsigma}_{k}\right)+B_{k} \underline{u}_{k+i-1} \\
& =A_{k}^{2} \underline{x}_{k+i-2}+A_{k} B_{k} \underline{u}_{k+i-2}+B_{k} \underline{u}_{k+i-1}+A_{k} \underline{\varsigma}_{k} \\
& \vdots \\
& =A_{k}^{i} \underline{x}_{k}+A_{k}^{i-1} B_{k} \underline{u}_{k}+\cdots+B_{k} \underline{u}_{k+i-1}+\left(A_{k}^{i-1}+\cdots+A_{k}+I\right) \underline{\varsigma}_{k}
\end{aligned}
$$

and therefore:

$$
\underline{y}_{k+i}=C_{k} A_{k}^{i} \underline{x}_{k}+C_{k} A_{k}^{i-1} B_{k} \underline{u}_{k}+\cdots+C_{k} A_{k}^{i-2} B_{k} \underline{u}_{k+1}+C_{k} B_{k} \underline{u}_{k+i-1}+D_{k} \underline{u}_{k+i}+\underline{d}_{i}
$$

with a determined vector:

$$
\underline{d}_{i}=C_{k}\left(A_{k}^{i-1}+\cdots+A_{k}+I\right) \underline{\boldsymbol{s}}_{k}+\underline{v}_{k}
$$

Now, if all predictive output vectors above $\underline{y}_{k+i}, 1 \leq i \leq N$ are rewritten as a mergence vector:

then it is obtained from (5):

$$
\underline{\boldsymbol{y}}=\operatorname{col}\left(\underline{y}_{k}, \underline{y}_{k+1}, \ldots, \underline{y}_{k+N}\right)
$$

$$
\underline{\boldsymbol{y}}=F \underline{p}+\underline{d}
$$

where: 


$$
\begin{aligned}
F & =\left(\begin{array}{ccccc}
D_{k} & \Theta & \cdots & \Theta & \Theta \\
C_{k} B_{k} & D_{k} & \cdots & \Theta & \Theta \\
\vdots & \vdots & \ddots & \vdots & \vdots \\
C_{k} A_{k}^{N-1} B_{k} & C_{k} A_{k}^{N-2} B_{k} & \cdots & C_{k} B_{k} & D_{k}
\end{array}\right), \\
\underline{p} & =\left(\begin{array}{c}
\underline{u}_{k} \\
\underline{u}_{k+1} \\
\vdots \\
\underline{u}_{k+N}
\end{array}\right), \underline{d}=\left(\begin{array}{c}
C_{k} \\
C_{k} A_{k} \\
\vdots \\
C_{k} A_{k}^{N}
\end{array}\right) \underline{x}_{k}+\left(\begin{array}{c}
\underline{d}_{0} \\
\underline{d}_{1} \\
\vdots \\
\underline{d}_{N-1}
\end{array}\right) \text { with } \underline{d}_{0}=\underline{v}_{k}
\end{aligned}
$$

and $\Theta$ denotes a zero matrix. It is easily to recognize, that the predictive mergence output vector $\underline{y}$ given in (6) depends only on all inputs $\underline{p}$ in the future associated in the current horizon $\left[t_{k}, t_{k+N}\right]$.

With the expression (6) of obtained predictive outputs $\underline{y}_{k+i}, 1 \leq i \leq N$, all tracking errors during the current control horizon will be deduced as follows:

$$
\underline{\boldsymbol{e}}=\underline{\boldsymbol{w}}-\underline{\boldsymbol{y}}=\underline{\boldsymbol{w}}-(F \underline{p}+\underline{d})
$$

where

$$
\underline{\boldsymbol{w}}=\operatorname{col}\left(\underline{w}_{k}, \underline{w}_{k+1}, \ldots, \underline{w}_{k+N}\right)
$$

is the mergence desired output values during the same control horizon.

Next, according to the output tracking purpose $\underline{y}_{k} \rightarrow \underline{w}_{k}$ or $\underline{\boldsymbol{e}} \rightarrow \underline{0}$ associated with the current control horizon, the mergence input vector $\underline{p}$ would be determined by minimizing the following objective function:

$$
\begin{aligned}
J_{k} & =\underline{\boldsymbol{e}}^{T} Q_{k} \underline{\boldsymbol{e}}+\underline{p}^{T} R_{k} \underline{p} \\
& =[\underline{w}-(F \underline{p}+\underline{d})]^{T} Q_{k}[\underline{w}-(F \underline{p}+\underline{d})]+\underline{p}^{T} R_{k} \underline{p} \\
& =\underline{p}^{T}\left(F^{T} Q_{k} F+R_{k}\right) \underline{p}-2(\underline{w}-\underline{d})^{T} Q_{k} F \underline{p}+(\underline{w}-\underline{d})^{T} Q_{k}(\underline{w}-\underline{d})
\end{aligned}
$$

which is obtained by replacing (8) into (10), or:

$$
J_{k}^{\prime}=\underline{p}^{T}\left(F^{T} Q_{k} F+R_{k}\right) \underline{p}-2(\underline{\boldsymbol{w}}-\underline{d})^{T} Q_{k} F \underline{p}
$$

since the last term $(\underline{w}-\underline{d})^{T} Q_{k}(\underline{w}-\underline{d})$ is independent on $\underline{p}$.

Since the objective function (11) is quadratic, the optimization problem:

$$
\underline{p}^{*}=\arg \min _{\underline{p} \in P} J_{k}^{\prime}(\underline{p})
$$

subjected to the constraint $p \in P$ with:

$$
P=\left\{\underline{p}=\operatorname{col}\left(\underline{u}_{k}, \ldots, \underline{u}_{k+N-1}\right) \in \mathbb{R}^{N m} \mid \underline{u}_{k} \in U\right\}
$$

can be solved by QP method, if the constraint $U$ is linear (described by linear equations or inequalities), or by SQP, if the constraint $U$ is nonlinear [3]. 
Finally, the input $\underline{u}_{k}$ for original perturbed nonlinear system (1) will be received from the optimal solution $\underline{p}^{*}$ of optimization problem (12) as follows:

$$
\underline{u}_{k}=(I, \Theta, \ldots, \Theta) \underline{p}^{*}
$$

This control value $\underline{u}_{k}$, which is clearly dependent on current system states $\underline{x}_{k}$ and therefore will be denoted afterward by $\underline{u}_{k}\left(\underline{x}_{k}\right)$, is only valid during the short current sampling time interval $t_{k} \leq t<t_{k+1}$. For determining the next control value $\underline{u}_{k+1}$ at the next time instant $k+1$ all calculation steps above, including (3), (7), (8)-(14), have to be repeated.

So, with (3), (7), (12) and (14) the state feedback controller $\underline{u}_{k}\left(\underline{x}_{k}\right)$ for nonlinear system (1), in which the system outputs belong current control horizon $[k, k+N]$ are predicted linearity, is established. However, it can be easily to recognize that since the minimizing of $J_{k}^{\prime}$ given in (11) occurs only over a finite control horizon $[k, k+N]$, the desired tracking performance $\underline{e}_{k}=\underline{w}_{k}-\underline{y}_{k} \rightarrow \underline{0}$ of this controller may not be satisfied. Therefore, to guarantee that the tracking error $\underline{e}_{k}$ always tends asymptotically to zero, an integral will be added supplementary to the proposed state feedback controller above.

Define two new variables:

$$
\Delta \underline{x}_{k}=\underline{x}_{k}-\underline{x}_{k-1}, \quad \Delta \underline{u}_{k}=\underline{u}_{k}-\underline{u}_{k-1}
$$

the LTI predictive model $H_{k}$ given in equation (4) will be changed to:

$$
H_{k}^{\prime}:\left\{\begin{array}{l}
\underline{z}_{k+1}=\widehat{A}_{k} \underline{z}_{k}+\widehat{B}_{k} \Delta \underline{u}_{k} \\
\underline{y}_{k}=\widehat{C}_{k} \underline{z}_{k}+D_{k} \Delta \underline{u}_{k} \text { for } t_{k} \leq t<t_{k+1}
\end{array}\right.
$$

where:

$$
\underline{z}_{k}=\left(\begin{array}{l}
\Delta \underline{x}_{k} \\
\underline{y}_{k-1}
\end{array}\right), \widehat{A}_{k}=\left(\begin{array}{cc}
A_{k} & \Theta \\
C_{k} & I_{r}
\end{array}\right), \widehat{B}_{k}=\left(\begin{array}{c}
B_{k} \\
D_{k}
\end{array}\right), \widehat{C}_{k}=\left(\begin{array}{ll}
C_{k} & I_{r}
\end{array}\right)
$$

and $I_{r}$ is the $r \times r$ identity matrix. This new LTI predictive model (16) has obviously an integral in it, because with:

$$
\operatorname{det}\left(\lambda I_{n+r}-\widehat{A}_{k}\right)=\operatorname{det}\left(\begin{array}{cc}
\lambda I_{n}-A_{k} & \Theta \\
-C_{k} & (\lambda-1) I_{r}
\end{array}\right)=(\lambda-1)^{r} \operatorname{det}\left(\lambda I_{n}-A_{k}\right)
$$

it has $r$ eigenvalues $\lambda=1$.

Finally, by using the new integral predictive model (16) instead of (4) to implement the state feedback controller $\underline{u}_{k}\left(\underline{x}_{k}\right)$, all matrices and vectors $A_{k}, B_{k}, C_{k}, \underline{x}_{k}, \underline{u}_{k}$ in operations (7), (14) will be replaced accordingly by $\hat{A}_{k}, \widehat{B}_{k}, \widehat{C}_{k}, \underline{z}_{k}, \Delta \underline{u}_{k}$ and $E, F, \underline{p}$ will be changed to:

$$
F=\left(\begin{array}{ccccc}
D_{k} & \Theta & \cdots & \Theta & \Theta \\
\widehat{C}_{k} \widehat{B}_{k} & D_{k} & \cdots & \Theta & \Theta \\
\vdots & \vdots & \ddots & \vdots & \vdots \\
\widehat{C}_{k} \widehat{A}_{k}^{N-1} \widehat{B}_{k} & \widehat{C}_{k} \widehat{A}_{k}^{N-2} \widehat{B}_{k} & \cdots & \widehat{C}_{k} \widehat{B}_{k} & D_{k}
\end{array}\right), E=\left(\begin{array}{c}
\widehat{C}_{k} \\
\widehat{C}_{k} \widehat{A}_{k} \\
\vdots \\
\widehat{C}_{k} \widehat{A}_{k}^{N}
\end{array}\right), \underline{p}=\left(\begin{array}{c}
\Delta \underline{u}_{k} \\
\Delta \underline{u}_{k+1} \\
\vdots \\
\Delta \underline{u}_{k+N}
\end{array}\right),
$$




\subsection{State observation}

To convert correlatively the state feedback controller $\underline{u}_{k}\left(\underline{x}_{k}\right)$ proposed above into an output feedback one $\underline{u}_{k}\left(\underline{\underline{x}}_{k}\right)$ based on separation principle, a suitable state observer:

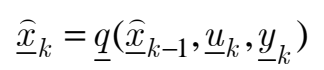

for the original nonlinear system (1) is required. In this paper the extended Kalman filter (EKF) and unscented Kalman filter (UKF) will be used for this purpose, because beside the state observation ability they have also an excellent behavior to filtering white noises $\underline{\zeta}_{k}, \underline{\xi}_{k}[4,5]$. All detailed calculations steps of EKF or UKF to obtain $\underline{x}_{k}$ from measurable signals $\underline{u}_{k}, \underline{y}_{k}$ ware already provided in $[4,5]$.

However, since the state vector $\underline{z}_{k}=\operatorname{col}\left(\Delta \underline{x}_{k}, \underline{y}_{k-1}\right)$ of the integral LTI predictive model $H_{k}^{\prime}$ given in (16) contains the system output in it, which is still disturbed by Non-Gaussian noise $\underline{d}_{k}$, and EKF/UKF can filter Gaussian noises $\underline{\boldsymbol{\zeta}}_{k}, \underline{\xi}_{k}$ only, this disturbance $\underline{d}_{k}$ must be eliminated first.

Denote the undisturbed output with $\underline{\hat{y}}_{k}=\underline{y}_{k}-\underline{\hat{d}}_{k}$, where $\underline{\hat{d}}_{k}$ is the mean of $\underline{d}_{k}$ over a certain observation horizon $M$, then is can be estimated averagely as follows:

$$
\underline{\hat{y}}_{k}=\underline{y}_{k}-\underline{\widehat{d}}_{k} \approx \underline{y}_{k}-\frac{1}{M} \sum_{i=0}^{M-1}\left(\underline{y}_{k-i}-\underline{g}\left(\underline{\widehat{x}}_{k-i}, \underline{u}_{k-i}, \underline{0}\right)\right)
$$

Finally, the filtering performance of EKF/UKF for original system (1) given above in (19) above will be changed accordingly to:

$$
\underline{\widehat{x}}_{k}=\underline{q}\left(\underline{\widehat{x}}_{k-1}, \underline{u}_{k}, \underline{\hat{y}}_{k}\right)
$$

\subsection{Output feedback control algorithm}

The following control algorithm summarizes all calculation steps given above to present completely the proposed output feedback controller.

1. Set $k:=0$. Choose arbitrarily initial values $\underline{x}_{-1}, \underline{x}_{-1}, \underline{\hat{y}}_{-1}$ and $N>M>0$.

2. Choose appropriately two symmetric positive definite matrices $Q_{k}, R_{k}$.

3. Set $\underline{x}_{k}=\widehat{x}_{k-1}$ and determine matrices $A_{k}, B_{k}, C_{k}, D_{k}$ according to (3), $\widehat{A}_{k}, \widehat{B}_{k}, \widehat{C}_{k}$ with (17), the vector $\underline{\boldsymbol{w}}$ with (9) and then $F, E$ according to (18).

4. Determine $\underline{p}^{*}$ of the optimal problem (12) by using QP or SQP algorithm, where the vector $\underline{x}_{k}$ in $J_{k}^{\prime}(\underline{p})$ given in (11) is replaced accordingly with $\underline{z}_{k}=\operatorname{col}\left(\Delta \underline{x}_{k}, \underline{y}_{k-1}\right)$.

5. Determine the control signal $\underline{u}_{k}=\underline{u}_{k-1}+\Delta \underline{u}_{k}=\underline{u}_{k-1}+\left(I_{m}, \Theta, \ldots, \Theta\right) \underline{p}^{*}$

6. Sent $\underline{u}_{k}$ to the original system (1) for a while of the sampling time interval $T$.

7. Measure the output $\underline{y}_{k}$ and then calculate $\underline{y}_{k}$ with (20).

8. Send $\underline{u}_{k}^{*}, \underline{\hat{y}}_{k}$ to EKF/UKF given in (21) for observation of $\underline{\widehat{x}}_{k}$.

9. Set $k:=k+1$ and go back to the step 2 . 


\section{SIMULATION RESULTS}

\subsection{Output Tracking Control of Inverted Pendulum}

Nowadays the inverted pendulum is considering as a fundamental benchmark in nonlinear control theory [6]. Hence, for an effective verifying of control performance of proposed controller it will be an adequate controlled object.

The inverted pendulum has a continuous time model as follows [6]:

$$
\begin{gathered}
\dot{x}=\underline{f}^{\prime}(\underline{x}, u)=\left(\begin{array}{c}
x_{2} \\
-\frac{\left(g \cos x_{3}-l x_{4}^{2}\right) m_{h} \sin x_{4}}{m_{c}+m_{h} \sin ^{2} x_{3}}+\frac{u}{m_{c}+m_{h} \sin ^{2} x_{3}} \\
x_{4} \\
\frac{\left(g-l x_{4}^{2} \cos x_{3}\right) m_{h} \sin x_{3}+g m_{c} \sin x_{3}}{l m_{c}+l m_{h} \sin ^{2} x_{3}}-\frac{u \cos x_{3}}{l m_{c}+l m_{h} \sin ^{2} x_{3}}
\end{array}\right) \\
\underline{y}=\underline{g}^{\prime}(\underline{x}, u)=\left(\begin{array}{l}
x_{1} \\
x_{4}
\end{array}\right)
\end{gathered}
$$

First, this model is converted in the discrete model (1) with sampling time $T=10^{-3} \mathrm{~s}$ :

$$
\underline{x}_{k+1}=\underline{x}_{k}+T \underline{f}^{\prime}\left(\underline{x}_{k}, u_{k}\right) \triangleq \underline{f}\left(\underline{x}_{k}, u_{k}\right) \text { and } \underline{y}_{k}=\underline{g}^{\prime}\left(\underline{x}_{k}, u_{k}\right) \triangleq \underline{g}\left(\underline{x}_{k}, u_{k}\right)
$$

Then by applying the proposed control algorithm with EKF for state observation and desired references as well as model parameters:

$$
\underline{w}=(0.5,1.5)^{T}, l=0,27[m], m_{h}=0,1[k g], m_{c}=1,2[k g], g=9,8\left[\mathrm{~m} / \mathrm{s}^{2}\right]
$$

two simulative system outputs $\underline{y}$ in the presence of both white noises $\underline{\varsigma}$, $\underline{\xi}$ have been obtained as exhibited in Fig. 2.

These obtained simulation results have indicated clearly a good output tracking performance of proposed controller as expecting. In spite of small tracking errors, but they are still acceptable for all systems with unstable equilibriums [6]. Moreover, these results also showed that the disturbances had been filtered effectively.
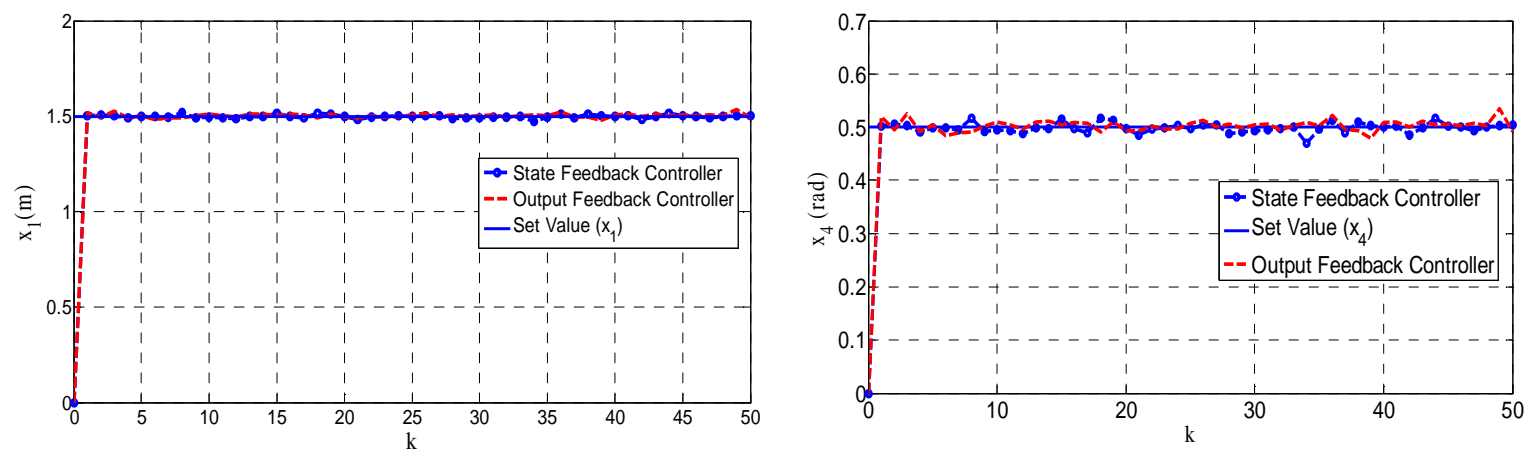

Figure 2. Simulation results of output tracking control of perturbed inverted pendulum. 


\subsection{Output Tracking Control of Boiler-Turbine System}

The boiler-turbine is an important device in thermal power plan, where the boiler creates stream by applying heat energy to water and then the steam spins a steam turbine to create electric power. The boiler-turbine unit has the following continuous time state model [7]:

$$
\begin{aligned}
& \underline{\dot{x}}=\underline{f}^{\prime}(\underline{x}, \underline{u})=\left(\begin{array}{c}
-0.0018 u_{2} x_{1}^{9 / 8}+0.9 u_{1}-0.15 u_{3} \\
\left(0.073 u_{2}-0.016\right) x_{1}^{9 / 8}-0.1 x_{2} \\
{\left[141 u_{3}-\left(1.1 u_{2}-0.19\right) x_{1}\right] / 85}
\end{array}\right) \\
& \underline{y}=\underline{g}^{\prime}(\underline{x}, \underline{u})=\left(\begin{array}{c}
x_{1} \\
x_{2} \\
0.05\left(0.13073 x_{3}+100 a_{c s}+q_{e} / 9-67.975\right)
\end{array}\right)
\end{aligned}
$$

where two model parameters:

$$
\begin{aligned}
& q_{e}=\left(0.854 u_{2}-0.147\right) x_{1}+45.59 u_{1}-2.514 u_{3}-2.096 \\
& a_{c s}=\frac{\left(1-0.001538 x_{3}\right)\left(0.8 x_{1}-25.6\right)}{x_{3}\left(1.0394-0.0012304 x_{1}\right)}
\end{aligned}
$$

are dependent on both system states $\underline{x}=\left(x_{1}, x_{2}, x_{3}\right)^{T}$ and system inputs $\underline{u}=\left(u_{1}, u_{2}, u_{3}\right)^{T}$.

Many effective control methods for boiler-turbine are available, but they are all essentially linear $[8,9]$. Hence, for their apply it is obligatory to linearize the model (23) around an equilibrium and which implies therefore the desired control performance could be guaranteed only in a neighborhood of this equilibrium.

To obtain the desired control performance over whole working space a nonlinear control method must be applied, for which the linearization of (23) do not be needed any more. The following simulation results for output tracking control of the boiler-turbine, depicted in Fig.3 and Fig.4, are obtained by applying the proposed nonlinear control algorithm for the discrete time system, which is received from (23) according to the discretizing equation (22) with $T=1 s$, together with UKF for state observation and:

$$
N=20, M=100, Q_{k}=\operatorname{diag}(2,80,0.2) \otimes I_{n}, R_{k}=I, \forall k
$$

as well as the required input constraints:

$$
0 \leq u_{i}[k] \leq 1, i=1,2,3 ; \quad(-0.007,-2,-0.05)^{T} \leq \Delta \underline{u}_{k}^{T} \leq(0.007,0.02,0.05)^{T}
$$

and the desired references, the output disturbances respectively as follows:

$$
\underline{w}=(129.6,105.8,0.64)^{T}, \underline{d}=(51.84,42.32,0.256)^{T}
$$

These simulation results exhibited in Fig. 3 and Fig.4 show, that all system outputs $y_{i}, i=1,2,3$ have converged asymptotically to their desired values $\underline{w}$, even there are both output disturbances $\underline{d}$ and white noises $\underline{\varsigma}, \underline{\xi}$ effect simultaneously to the boiler-turbine system (23). Furthermore, Fig.4 on the left site also indicated that the required input constraints had been satisfied additionally. In other words, the obtained simulation results have asserted an excellent output tracking performance and disturbance attenuation behavior of proposed control algorithm. 

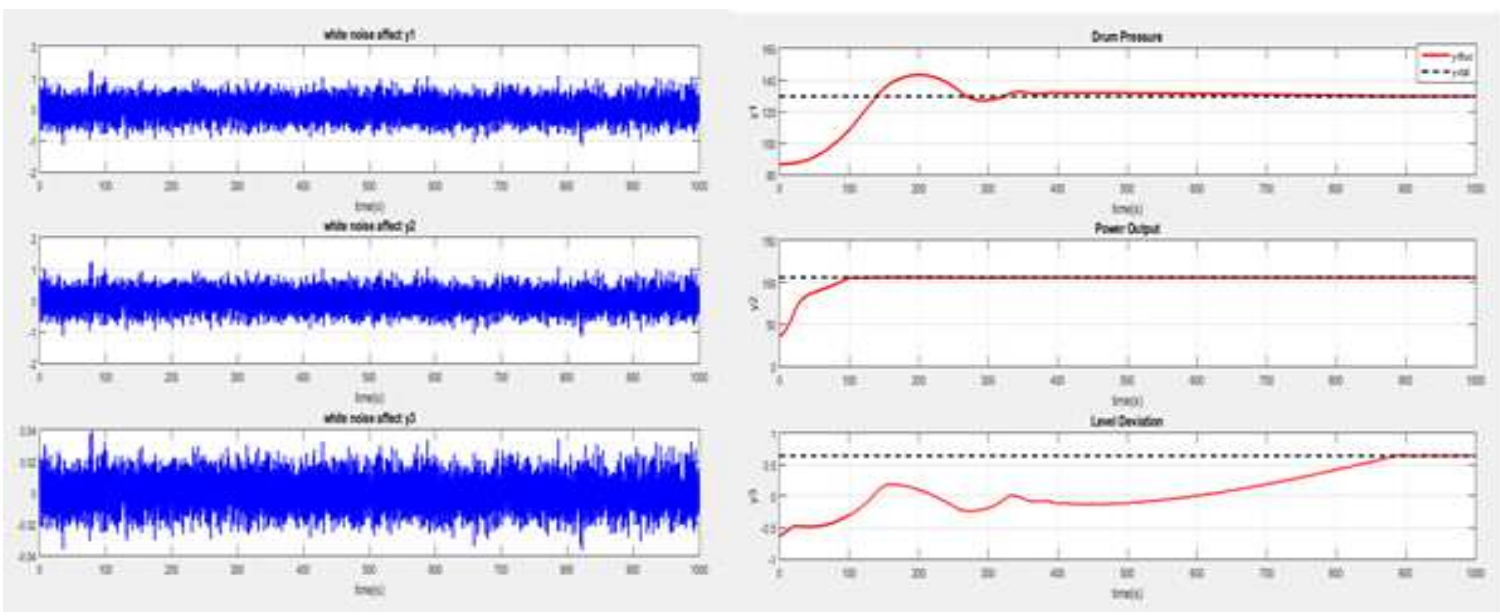

Figure 3. System responses in presence of white noises $\underline{\varsigma}, \underline{\xi}$.
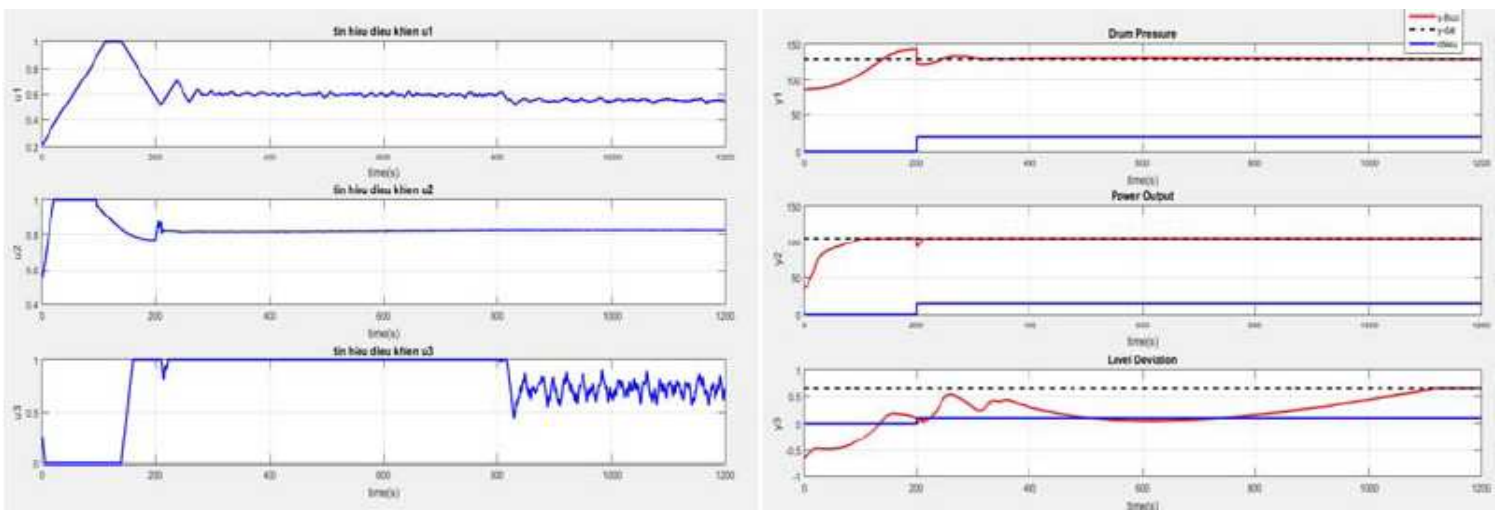

Figure 4. Constrained inputs and responses of closed loop boiler-turbine system in presence of both white noises $\underline{\underline{S}}, \underline{\xi}$ and output disturbances $\underline{d}$.

\section{CONCLUSIONS}

The paper has proposed an output feedback controller to constrained output tracking control nonlinear systems. This controller is established by combining an appropriate constrained state feedback controller with a suitable state observer, which can filter additionally noises and output disturbances in systems.

The simulation results obtained by applying this controller to constrained output tracking control inverted pendulum and boiler-turbine unit in a thermal power plan have showed, that the proposed controller could be applied also for a wide range of perturbed nonlinear systems.

\section{REFERENCES}

1. Maciejowski M. J. - Predictive control with constrains, Prentice Hall, 2011.

2. Grüne L. and Pannek J. - Nonlinear model predictive control, Theory and Algorithms, Springer, 2010.

3. Nocedal J. and Wright S. J. - Numerical Optimization. Springer-New York, 1996. 
4. Grewal M. S. and Andrews A. P. - Kalman filtering: Theory and Practice using MatLab. John Wiley \& Sons, (2001).

5. Julier S. J. and Uhlmann J. K. - Unscented filtering and nonlinear estimation. Proceedings of the IEEE 92 (3) (2004) 401-422.

6. Boubaker O. - The Inverted Pendulum Benchmark in Nonlinear Control Theory - A Survey, Int. Journal of Advanced Robotic Systems. ISSN 1729-8806, May 7, 2013

7. Bell R. D., Åström K. J. - Dynamic Models for Boiler Turbine Alternator Units: DataLogs and Paramter Estimation for 160MW Unit. Lund Institute of Technology, TRFT-3192, (1987).

8. Xiao Wu, Jiong Shen, Yiguo Li - Control of Boiler-turbine Coordinated System Using Multiple-model Predictive Approach. 2010 8th IEEE International Conference on Control and Automation Xiamen, China, June 9-11, 2010.

9. Zhang S., Taft CW., Bentsman J., Hussey A., Petrus B. - Simultaneous gains tuning in boiler/turbine PID-based controller clusters using iterative feedback tuning methodology. ISA Trans. 51 (2012) 609-21. 